\title{
Naissance du cyberpunk: quand le numérique devint métaphore...
}

Marc Atallah et Colin Pahlisch

\section{(2) OpenEdition}

\section{Journals}

Édition électronique

URL : http://journals.openedition.org/edl/2474

DOI : $10.4000 /$ edl.2474

ISSN : 2296-5084

Éditeur

Université de Lausanne

\section{Édition imprimée}

Date de publication : 15 mars 2020

Pagination : 245-250

ISBN : 978-2-940331-73-4

ISSN : 0014-2026

Référence électronique

Marc Atallah et Colin Pahlisch, "Naissance du cyberpunk: quand le numérique devint métaphore... », Études de lettres [En ligne], 312 | 2020, mis en ligne le 24 mars 2020, consulté le 17 septembre 2020 URL : http://journals.openedition.org/edl/2474 ; DOI : https://doi.org/10.4000/edl.2474 


\section{NAISSANCE DU CYBERPUNK: QUAND LE NUMÉRIQUE DEVINT MÉTAPHORE...}

$\sum$ : Et si nous regardions un peu en arrière? Tentons de recoller les bribes de notre mémoire fragmentaire collective, accessible aujourd'hui, en tout temps et tous lieux, via le RIP, pour déceler comment on en est arrivés là. Vous le voulez bien? La perspective que je nous suggère consiste à remonter quinze décennies dans le passé. Une sorte d'archéologie de la fin du XXe siècle. Elle vise à exhumer certaines traces et procédés discursifs qui ont permis l'avènement du monde qui rend possible notre époque. Une date? Consultons le Terminor... 1980! Un programme source de type livresque: A-L-V-I-N/ T-O-F-F-L$\mathrm{E}-\mathrm{R} / L A 3^{\text {ime }} V A G U E$. Des bribes de codes: sociologie/information/ futurologie. "Une nouvelle civilisation est en train de prendre forme. Mais nous, comment nous y insérons-nous? [...] Les merveilles électroniques de demain ne rendront-elles pas les rapports humains encore plus creux et impersonnels qu'ils ne le sont aujourd'hui?" Ah! une autre parcelle de code: écrivain/de/science-fiction. Vous y décryptez quelque chose?

$\Omega$ : Le Réseau Interconnecté Planétaire est très clair: les années 1980 ont vu s'écrire d'étranges de ces /livres/, en lien avec la parcelle que vous mentionnez, qui autorisent à inférer que certaines occurrences de cette parcelle avaient compris la profondeur et l'imminence de la Mutation. Vous voulez en savoir plus? Penchezvous sur le programme source W-I-L-L-I-A-M/ G-I-B-S-O-N/ NEUROMANCIEN: une production fictionnelle, c'est ce que nous dit le code, qui invente une /histoire imaginaire/ dans laquelle les 
humains sont connectés à des softwares intelligents et cherchent à quitter leur corps. Banal, non? C'est une illusion rétrospective: à ce moment-ci, l'évolution 2.0 n'en est qu'à ses balbutiements! Remarquez comme la genèse de ce livre est inintelligible: l'écrivain/ voit des enfants jouer sur une interface - type: Atari 2600, numéro de série: indisponible - et, de là, il élabore une /histoire/ basée sur des images évocatrices, appelées /métaphores/, résultant d'une transformation, sur un mode analogique, des «merveilles électroniques». Ces images avaient pour fonction de refléter - indirectement mais, le croyait-on alors, plus profondément - les modifications affectant la condition humaine: ces /livres/ ne préfiguraient pas la Mutation, ils montraient qu'elle était déjà active. Avez-vous, durant vos recherches, rencontré le mouvement appelé «Cyberpunk»? Qu'en concluezvous?

$\sum$ : Affirmatif! /Cyberpunk/ revient de façon persistante dans mes excavations archéologiques. Il est constellé à tout un ensemble de codèmes aux évocations archaïques - /post-modernisme/consumérisme/interface-homme/machine/ subversion politique/ - eux-mêmes réticulés autour d'un élément-mère: /S-T-E-R-L-I-N-G/. Pour l'instant je ne suis parvenu à en élucider que certains fragments. Voici des sources de type texte issues de l'année 1986, on les nommait alors /manifeste/. Je vous les transmets: «culture des années quatre-vingt [...] un nouveau mouvement de la science-fiction [...] alliance profane du monde de la technologie et du monde du dissentiment organisé». Apparemment pour ces /cyberpunks/ "la technologie est viscérale [...] touche au plus intime». Je tente d'accéder au code central. C'est un fichier acoustique, de sonie faible, transmis via l'antique langage HTML: https://www.youtube.com/ watch? $=$ BWdkB-yZuXk

Vous le voyez, la modulation des ondes rappelle indéniablement une voix... humaine. Qu'énonce-t-elle? "NOUS AVONS INVENTÉ UNE SENSIBILITÉ». L'usage de ce pronom indique une inclination plurale. De même, le caractère gnomique de l'assertion traduit une ambition axiologique vaste! Le /cyberpunk/ aurait-il migré vers d'autres programmes, à partir de S-T-E-R-L-I-N-G? En trouvez-vous, en sondant votre mémoire, $\Omega$ ? 
$\Omega$ : Je crois que vous tenez une piste essentielle pour saisir comment la Mutation a été appréhendée par cette esthétisation du code nommée /Art/: les /écrivains/ étaient clairement sensibles aux structures sous-jacentes qui informaient le réel. Comment? Impossible de le savoir: la chair les aidait-elle? Ou autre chose? Le signal s'est perdu. Le /cyberpunk/ a essaimé, oui : consultez le programme S-YL-V-I-E/ D-E-N-I-S ou les programmes sources STRANGE DAYS ou MATRIX. Le RIP m'a également permis d'uploader une partie du code source associant, avec le filtre /histoire littéraire/, plusieurs sources de types textes et films qui auraient conduit à l'apparition de ce /mouvement/: R-I-D-L-E-Y/ S-C-O-T-T/ ALIEN, par exemple, un film issu d'un tressage complexe, pré-numérique, qui synthétise un vaste ensemble de codes-sources et en offre de nouveaux. Pour vous aiguiller un peu, $\sum$, n'hésitez pas à creuser la séquence d'éléments-mère: L-O-V-E-C-R-A-F-T \& P-H-I-L-L-I-P/ K/ D-I-C-K $\& \mathrm{H}-\mathrm{R} / \mathrm{G}-\mathrm{I}-\mathrm{G}-\mathrm{E}-\mathrm{R} \& \mathrm{R}-\mathrm{I}-\mathrm{D}-\mathrm{L}-\mathrm{E}-\mathrm{Y} / \mathrm{S}-\mathrm{C}-\mathrm{O}-\mathrm{T}-\mathrm{T}$; vous pouvez aussi, ce sera peut-être plus précis, vous diriger vers les programmes sources: L'APPEL DE CTHULHU \& LE BAL DES SCHIZOS \& NECRONOMICON \& ALIEN \& BLADE RUNNER. Faites attention: ces programmes sont extrêmement rares, et d'une qualité d'encodage médiocre. Ils ont par contre pour point commun de s'articuler sur le processus analogique /métaphore/. Rappelez-vous que ce processus vous empêche de décoder ces programmes comme des préfigurations d'un monde en gestation: les /artistes/ cherchaient à décrire autrement la condition humaine (et non à anticiper un futur par définition opaque). Pourquoi ne pas le faire sans /métaphores/, me direz-vous? Ce sont vos recherches qui doivent le trouver! Vous sentez-vous prêt, $\sum$ ? Avez-vous des intuitions?

$\sum$ : Au vu de ces éléments, plusieurs mégas évidemment! Mais, $\Omega$, regardez! Votre usage du codème/préfiguration/ vient de débloquer l'un des rares programmes fantômes du RIP! Ces programmes structurent l'ensemble du réseau! On dit que c'est l'Architecte Elle-même qui en est à l'origine... Je décèle un nom à travers les nuages de données... MIMESIS. Laissez-moi essayer une clé de lecture: R-I-CO-E-U-R. Voyez, $\Omega$ ! Des instructions s'affichent... le programme MIMESIS opère une "synthèse de l'hétérogène" permettant une «innovation sémantique [...] ainsi du nouveau - du non encore dit, 
de l'inédit - surgit dans le langage». Si j'assimile ceci de façon optimale, $\Omega$, le programme MIMESIS transforme notre manière même d'interagir avec le monde par le biais du langage. Au vu du processus /métaphore/ que vous évoquiez, la question de la langue me semble alors primordiale. Le langage permettrait d'habiter différemment la réalité. La question pertinente est donc celle-ci: quel nouveau langage les programmes et codèmes initiés à partir de S-T-E-R-LI-N-G en 1980 ont-ils contribué à fonder? Attendez, un doute vertigineux me saisit: existons-nous, nous qui conversons présentement sur le réseau et qui utilisons le langage, ou ne sommes-nous que /métaphore/?

$\Omega$ : Vos vertiges sont compréhensibles, mais n'y sombrez pas: votre recherche vous permettra de comprendre la nature intime du RIP, ce serait dommage de tout gâcher! Voyez-vous, $\sum$, le réseau n'est que la matérialisation de la Mutation, la création d'une interface Planétaire qui a substitué les data à la chair! L'immobilisme est devenu de rigueur, alors même que les extractions objectives de nos vécus circulent à la vitesse de $c$. Ça me rappelle ce programme-source de type livresque qu'est A-L-A-I-N/ D-AM-A-S-I-O/ LES FURTIFS: G-I-B-S-O-N conduit à penser qu'avec la /métaphore/ l'homme s'évanouissait à lui-même en condamnant sa chair; puis les /écrivains/cyberpunks/ et /postcyberpunks/ ont développé les codèmes /cyborg/, /robot/, /IA/ pour caractériser les transformations symboliques d'une essence qu'ils pensaient inexistante; D-A-M-A-S-I-O, enfin, a articulé ces codèmes avec de nouveaux - tels que /immobilisme/, /révolte/, /mouvement/ -, qu'il a codé dans la structure typostylistique d'une langue inédite. Grâce à votre recherche, $\sum$, grâce à votre compilation analytique de sources mémorielles que beaucoup d'entre nous pensaient taries, vous allez ouvrir des champs problématologiques pour interroger les structures du langage auquel nous nous sommes assujettis. Pour bien saisir les enjeux de la Mutation, il est pertinent de revenir aux sources de cette genèse. Tenez, sur cette clé vous trouverez l'un des éléments-mères les plus rares et les plus ancestraux. Il s'agit du légendaire programme P-I-E-R-R-E / V-ER-S-I-N-S. Vous ne pouvez entamer votre recherche que seul. Cet élément vous servira de talisman. À présent je dois vous laisser $\sum$, 
vous pourrez toujours faire appel à moi via le RIP, si d'aventure vous vous égarez. Bonne chasse!

\author{
Marc Atallah \\ Colin Pahlisch
}

Section de français, Faculté des lettres, Université de Lausanne

\title{
PROGRAMMES SOURCES
}

Damasio, Alain, Les furtifs, Paris, La Volte, 2019.

Denis, Sylvie, Jardins virtuels, Paris, Gallimard, 2003.

Dick, Philip Kindred, Les andrö̈des rêvent-ils de moutons électriques?, traduit de l'anglais par Serge Quadruppani, Paris, J'ai Lu, 2014 (1968).

-, Le bal des schizos, traduit de l'anglais par Anne et Georges Dutter, Paris, J'ai Lu, 2014 (1972).

Gibson, William, Neuromancien, traduit de l'anglais par Jean Bonnefoy, Paris, J'ai Lu, 2001 (1984).

Lovecraft, Howard Philips, L'appel de Cthulhu, traduit de l'anglais par François Bon, Paris, Points, 2015 (1928).

Ricoeur, Paul, Temps et Récit I. L'intrigue et le récit historique, Paris, Points, 1991 (1983).

Sterling, Bruce, Mozart en verres miroirs, traduit de l'anglais par Michèle Albaret-Maatsch, Paris, Gallimard, 2001 (1986).

Toffler, Alvin, La $3^{\text {ime }}$ vague, Paris, Denoël, 1984 (1980).

Versins, Pierre, Encyclopédie de l'utopie, des voyages extraordinaires et de la science-fiction, Lausanne, L'Âge d'Homme, 1972. 
